\title{
Planificación estratégica situacional en la gestión escolar de las instituciones educativas
}

\section{Situational strategic planning in the school management of educational institutions}

\author{
Roberto Tito Trinidad Marcelo*1 \\ *Departamento Académico de Educación, Facultad de Educación, Universidad Nacional del Centro del Perú (UNCP), Huancayo - Perú.
}

\begin{abstract}
Resumen
El presente artículo está basado en la metodología de la Investigación Acción, en el análisis documentario de los instrumentos de gestión del proceso de la gestión escolar en las instituciones educativas públicas y del proceso de planificación, para ello centrándonos en la planificación estratégica situacional de Carlos Matus. Se puede ver que la carencia y la diversidad de modelos empleados en la formulación de instrumentos de gestión en las instituciones educativas no permite la consecución del objetivo institucional deseado, solo por avocarse al cumplimiento documentario de momento, esto se repite cada año, acción que no se refleja en resultados tal como se espera por ser un objetivo nacional que es elevar el nivel académico, que como parte implícita está la gestión escolar, también se refleja la falta de articulación de los instrumentos de gestión institucional con la gestión pedagógica, administrativa y en algunos aspectos con los objetivos estratégicos regionales y nacionales, para lo cual se analizan los procesos de la gestión educativa, a fin de determinar la pertinencia del modelo de la planificación estratégica situacional aplicado a la gestión escolar.
\end{abstract}

Palabras Claves: Planificación, estrategia, gestión, educativa, objetivos.

\begin{abstract}
This article is based on the methodology of Action Research, on the documentary analysis of the management instruments of the school management process in public educational institutions and of the planning process, for this, focusing on the strategic situational planning of Carlos Matus (Matus, 1977). We can see that the lack and diversity of models used in the formulation of management instruments in educational institutions does not allow the achievement of the desired institutional objective, only by advocating document compliance for the time being, this is repeated every year, an action that is not reflects in results as expected as it is a national objective that is to raise the academic level, which as an implicit part is school management, it also reflects the lack of articulation of institutional management instruments with pedagogical, administrative and in some aspects with the regional and national strategic objectives, for which we analyze the educational management processes, in order to determine the relevance of the situational strategic planning model applied to school management.
\end{abstract}

Keywords: Planning, strategy, management, educational, objectives.

1 Correspondiencia: Roberto Tito Trinidad Marcelo, rotrima2@gmail.com 


\title{
Planificação estratégica situacional na gestão escolar das instituições educativas
}

\begin{abstract}
Resumo
O presente artigo está baseado na metodologia da Investigação ação, na análise documentária dos instrumentos de gestão do processo da gestão escolar nas instituições educativas públicas e do processo de planificação, para ele centralizamos na planificação estratégica situacional de Carlos Matus (Matus, 1977). Podemos ver que a carência e a diversidade dos modelos empregados na formulação de instrumentos de gestão nas instituições educativas não permite a aquisição do objetivo institucional desejado, somente por afastar-se ao cumprimento documentário do momento, isto se repete a cada ano, ação que não se reflete nos resultados como tal espera por ser um objetivo nacional que é elevar o nível acadêmico, que como parte implícita está na gestão escolar, também se reflete na falta de articulação dos instrumentos de gestão institucional com a gestão pedagógica, administrativa e em alguns aspectos com os objetivos estratégicos regionais e nacionais, para o qual analisamos os processos da gestão educativa, a fim de determinar a pertinência do modelo da planificação estratégica situacional aplicado para a gestão escolar.
\end{abstract}

Palavras claves: Planificação, estratégia, Gestão, educativa, objetivos.

\section{Allin ruana estratégica situacional kan chay gestionpi llapallan institucionpi educativapi}

\section{Uchuycha}

Kayarticulumkam metodología de la investigación acción nisqampi chay kuskinaqillqarina chay gestión del proceso de la gestión escolar llapallanyachaywasimanestadupa chal planificación risqan, chaymi ruwaspa planificacionta estratégica situacional chay Carlos Matus ruwasqanmanta Ricunchimmanakasqantainaspaachka modelos ruwasqa chay formulación de instrumentos de gestión llapallanyachaywasipawallpachaykunata mana munanchuqatipayta chay objetivo institucional munasqata, achkaruway Kamasqantadocumentukunata, watawatanchayllatakutinruray, ankay mana lluqsinchumunasqahinatakaptinhatunrirayyuyayhananchaqhatun yachayta, kaptin gestión yachaywasiman, chaymi mana rikukunchu articulación llapallan instrumento chay gestión wallpachay chay gestión pedagógica administrativa chay hukkunaaspectukunatukukuyestratégicos regionales tukuy mama llaqtapi, chaymiamutaykuspaprocesoskunata chay gestión educativapata, allintaruwanapaq chay modelo planificación estratégica situacional Ruwaspaqunanchikpaq chay gestión escolarman.

Hatun apup simi: Allin ruana, estrategicam, Gestionmi, educativan, tukukuy. 


\section{Introducción}

Muchas instituciones educativas públicas viven su propia problemática interna, carentes de trabajo en equipo, objetivos y metas inalcanzables, no se evidencian respuestas en lugar de actuar proactivamente como correspondería falta de capacidad de reunir gente pensante, y los cambios que se van dando a pasos agigantados en este mundo tecnológico y globalizado y como organización actuamos como carentes de la suficiente agilidad mental que les permita elaborar un pensamiento dinámico y flexible, no se demuestra respuestas oportunas y efectivas, un proceso de gestión escolar con mucha debilidad, por lo tanto una planificación estratégica adecuada es la que debe encaminar hacia donde quieren llegar y cuál es el servicio educativo que deben brindar estas instituciones a fin de optimizar los recursos y la gestión hacia un resultado.

Los instrumentos de gestión de la institución educativa (Proyecto Educativo Institucional - PEI, el Plan Anual de Trabajo - PAT, el Proyecto Curricular de la Institución - PCI y el Reglamento Interno - RI) tienen el propósito de orientar su gestión hacia el logro de su objetivo, a un futuro deseado, que es básicamente elevar el logro de los aprendizajes, el acceso y la permanencia de los estudiantes a través del liderazgo pedagógico de sus directivos; para Matus, C. (1977), el análisis de la situación problemática es la guía de acción para poder generar una planificación estratégica situacional, asume la crítica de la planificación tradicional, siendo referencia del "hombre de acción", que debe resolver los problemas más complejos, mostrando entonces, una herramienta de conducción o gobierno del proceso social en todas sus dimensiones, está integrada de un enfoque metodológico debido al desarrollo de categorías centrales que, con sus interrelaciones, son capaces de representar las relaciones direccionales y causales de una tipología de problemas que se suponen objeto de intervención social.

El enfoque cualitativo que empleamos; está ligado exclusivamente a una determinada forma de hacer investigación tal como menciona Vasilachis, I. (2006). Así mismo la metodología investigación acción nos ayuda al estudio de la problemática situacional de gestión escolar en las instituciones educativas, para Martínez Miguelez, el método de la investigación acción implica una nueva visión del hombre y de la ciencia, los sujetos investigados son auténticos coinvestigadores, participando muy activamente en el planteamiento del problema a investigar. Martínez, M. (2004 p.239)

\section{Desarrollo de las variables}

\section{Concepto de Investigación Acción}

La planificación estratégica situacional, es un modelo de investigación acción orientado a una planificación que establece metas y objetivos que deben ser cumplidos durante periodos específicos, resolviendo problemas complejos dentro de una gestión escolar: identificación del problema trascendente, análisis del problema, formulación del plan de acción (objetivos, acciones, tiempo, estrategia, canales, responsabilidades, recursos e indicadores), recolección de la información necesaria, categorización de información, estructuración de las categorías, ejecución de un plan de acción, evaluación de la acción ejecutada y propuesta estética emergente. Inicia desde el análisis del contexto de la gestión, identificando y describiendo la situación problemática, para luego diseñar el objetivo, la gran visión, misión actual y futura y el diseño estratégico para su operativización.

\section{Diseño General del Proyecto}

El proyecto de investigación acción se diseñó utilizando el modelo del planeamiento estratégico situacional. Es una herramienta de gestión que apoya la toma de decisiones en toda organización, tiene por finalidad afianzar y obtener eficiencia y eficacia en el manejo de los instrumentos de gestión. 


\section{Diseño Identificación del Problema Trascendente}

Es el desconocimiento del modelo y su proceso de la planificación estratégica situacional, el diagnóstico FODA no es articulado eficientemente para priorizar la problemática institucional, dificultando en el logro de los objetivos.

\section{Análisis del Problema}

La gran mayoría de las instituciones educativas públicas formulan y articulan de manera deficiente los instrumentos de gestión (PEI, PAT, PCI y RI). Ya que ello tiene el propósito de orientar su gestión hacia el logro de su objetivo que es básicamente elevar el logro de los aprendizajes, el acceso y la permanencia de los estudiantes a través del liderazgo pedagógico de sus directivos; para una toma de decisiones oportuna y pertinente y lograr el futuro deseado.

\section{Formulación del Plan de Acción}

Las causas más comunes en una gestión escolar sin la planificación estratégica situacional son: poco involucramiento de los integrantes de la comunidad educativa al elaborar el PEI; al formular la visión y misión no se toma en cuenta el diagnóstico real de la institución educativa, elaborado solo por cumplir; se realiza una planificación estratégica ineficiente, objetivos y metas inalcanzables. Por lo que el planeamiento estratégico situacional ayudará a formular de manera eficiente.

Las consecuencias que se asumirán a la falta de la planificación estratégica situacional serán; los trabajos pedagógicos no serán direccionados a la propuesta de gestión centrada en los aprendizajes, la visión, objetivos y metas son propuestas inalcanzables, estancamiento institucional y no logrando el futuro deseado.

\section{Plan de Acción}

\section{Objetivos}

Lograr que la planificación estratégica situacional sea un modelo de planeamiento en una gestión escolar en la que resuelve problemas emergentes del contexto.

\section{Acciones}

Organizar el equipo de planeamiento, con actores empoderados de la problemática institucional.

Conocimiento del modelo de planeamiento estratégico situacional.

Aplicación del proceso metodológico de planeamiento estratégico situacional basado en la problemática institucional y su resolución de la misma.

Conocimiento de los procesos de la gestión escolar y su articulación con los demás instrumentos de gestión; según Loera (2003) señala que; la gestión escolar es el conjunto de labores realizadas por los actores de la comunidad educativa.

\section{Tiempo}

El periodo que debe regir el proceso de formulación aplicando la planificación estratégica situacional es durante los meses de enero y febrero, y las actividades operativas plasmadas en el Plan Anual de Trabajo es de cumplimiento durante el desarrollo académico. 


\section{Estrategia}

La estrategia empleada será; la formulación de la visión, misión, metas, objetivos; las actividades operativas deben ser alcanzables, realizables y articulados, culminado el periodo debe ser evaluado y sociabilizado con los actores educativos para la toma de decisiones según el modelo de la planificación estratégica situacional, dentro de la gestión escolar (Matus, 1967).

\section{Canales}

La acción del planeamiento estratégico situacional se realizará durante el desarrollo de la gestión escolar.

\section{Responsabilidades}

A fin de consolidar una correcta aplicación del modelo de planificación estratégico situacional, la responsabilidad recae en la comisión de formulación de los instrumentos de gestión y su ejecución y cumplimiento recae en el director y directivos que dirigen la gestión escolar. Por lo tanto "la dirección es el elemento del proceso administrativo que tiene como finalidad coordinar los elementos humanos de las empresas, implica que un responsable con nivel de autoridad genere liderazgo, así como motivación, comunicación, cambio organizacional e individual y creatividad". (Ruiz, 2012, p. 11)

\section{Recursos}

Los recursos empleados serán; el recurso humano, y materiales para su consolidación y difusión.

\section{Indicadores}

- Las acciones pedagógicas serán centradas en los aprendizajes y demostradas en una evaluación.

- La visión, objetivos y metas son propuestas alcanzables y evaluables al cierre del año académico.

- Gestión escolar eficaz y eficiente, resuelve problemas emergentes.

- Instrumentos de gestión escolar debidamente articulados.

\section{Recolección de la Información Necesaria}

La información que se está evaluando corresponde al cuestionario resuelto por directores de los tres niveles de la gestión escolar; nivel inicial, primario y secundario de la educación básica regular. Planteando interrogantes que respondan al objeto de la investigación; con la finalidad de evaluar que la planificación estratégico situacional sea un modelo de planeamiento en una gestión escolar en la que resuelve problemas emergentes del contexto.

\section{Categorización de Información}

Se inicia con reuniones generales de la comunidad educativa, realizando el diagnóstico institucional, el análisis FODA para fundamentar la razón del servicio educativo, como está ubicado y plantear la misión, visión, objetivos y valores institucionales a un futuro deseado. De ello se deriva las acciones operativas plasmadas en el PEI, PCI, PAT y RI para cumplir en la gestión escolar y la comunidad educativa. Su redacción debe estar en tiempo presente claro, preciso y alcanzable proyectado a tres años.

\section{Estructuración de las Categorías}

Se estructura los procedimientos de las categorías considerando los aspectos fundamentales hasta su ejecución para lo cual se diagrama: 


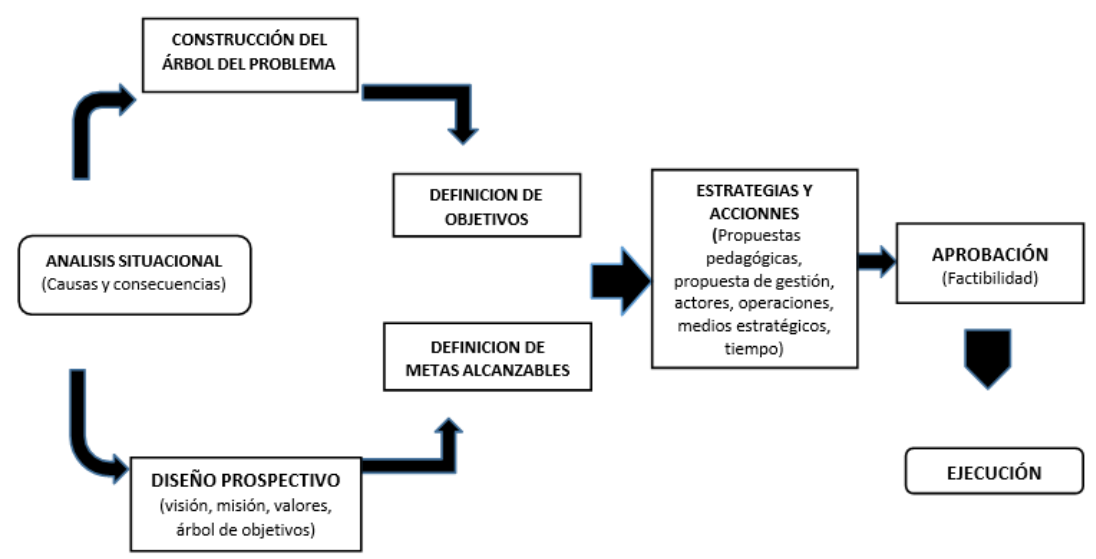

Figura 1. Estructura de categorización.

\section{Ejecutar la propuesta}

Se inicia conformando el equipo de planeamiento en la institución, conocer el modelo de planeamiento estratégico situacional es fundamental para su formulación de los instrumentos de gestión, se analiza la situación problemática de la institución que es guía de acción; se plantea el objetivo que es "lograr que la planificación estratégico situacional sea un modelo de planeamiento en una gestión escolar en la que resuelve problemas emergentes del contexto".

Las acciones a plantear son el proceso metodológico de planeamiento estratégico situacional basado en la problemática institucional y su resolución de la misma; conocimiento de los procesos de la gestión escolar y su articulación con los demás instrumentos de gestión; este trabajo se desarrolla a inicio del proceso académico y las actividades operativas es de cumplimiento durante el año.

Las estrategias empleadas fueron; la formulación de la visión, misión, metas, objetivos y actividades operativas alcanzables, realizables y articulado con los demás instrumentos de gestión DISEÑO PROSPECTIVO (Visión, misión, valores y árbol de objetivos), APROBACIÓN (Factibilidad), DEFINICIÓN DE OBJETIVOS, DEFINICIÓN DE METAS ALCANZABLES, ESTRATEGIAS y ACCIONES (Propuestas pedagógicas, propuesta de gestión, actores, operaciones, medios estratégicos y tiempo), CONSTRUCCIÓN DEL ÁRBOL DEL PROBLEMA, EJECUCIÓN y ANÁLISIS SITUACIONAL (Causas y consecuencias) escolar; sometiéndolo luego a ser evaluado y sociabilizado con los actores educativos; garantizándonos de esta manera un trabajo eficiente.

Los responsables de la formulación de los instrumentos de gestión recaen en el equipo de planeamiento, su ejecución y cumplimiento está a cargo del director y directivos que dirigen la gestión escolar; esto nos garantiza su consolidación de las actividades operativas.

Se culmina en el análisis de los indicadores, que son; las acciones pedagógicas serán centradas en los aprendizajes y demostrados en una evaluación, la visión, objetivos y metas son propuestas alcanzables y evaluables al cierre del año académico, una gestión escolar eficaz y eficiente que resuelve problemas emergentes e instrumentos de gestión escolar debidamente articulados; los resultados de estos indicadores nos demostrarán su logro o deficiencia.

\section{Evaluación de la acción ejecutada}

A través de un cuestionario sometido a los actores de la gestión escolar, pudimos observar que, la aplicación de la planificación estratégica situacional, resuelve diversos problemas emergentes de una gestión escolar, el futuro deseado de la institución, la consolidación de los objetivos y metas, la articulación de los instrumentos de gestión, actividades operativas que responden a los objetivos planteados mediante las estrategias, son acciones ejecutadas que nos garantizan el uso de este modelo de planificación. 
La investigación acción utilizada como metodología sirvió para mi participación muy activa en la investigación como organizador, como facilitador del proceso y en general como un técnico y recurso disponible, tal como indica Martínez (2004).

\section{Propuesta estética emergente}

Luego de la experiencia en la aplicación de la planificación estratégica situacional en la gestión escolar, mostrando resultados que responde a la necesidad institucional en su proceso de gestión escolar, se propone su aplicación, porque también apoya a la articulación del proyecto educativo institucional, plan anual de trabajo, reglamento interno, proyecto curricular de la institución, orientado al cumplimiento de los objetivos y metas planteado, enmarcadas en los enfoques de gestión, centrada en los aprendizajes y considerando las necesidades de los estudiantes, con metas alcanzables en el periodo planteado.

\section{Validez y confiabilidad}

En la presente investigación, los datos se han recogido de los mismos actores investigados, personas que están desarrollan el proceso de la gestión escolar, convirtiéndose para nosotros en coinvestigadores, dando el rigor y la seguridad en la conclusión que se llegaron, debido a que se captaron cada evento desde las diferentes etapas del desarrollo de la planificación, participando de manera proactiva hasta la etapa final del proceso de la ejecución y evaluación, convirtiéndolo de esta manera válida y confiable el instrumento utilizado y los datos analizados.

\section{Conclusiones}

Finalmente concluimos la presente investigación considerando aspectos que ayudan a un correcto planeamiento en la gestión escolar:

Un análisis situacional problemática eficiente de la gestión institucional ayudará de manera efectiva para la formulación y planteamiento del objetivo y meta institucional, y de manera articulada entre los diversos instrumentos de gestión a ser cumplida en el tiempo establecido.

La metodología y el procedimiento de la planificación estratégico situacional que se aplicó, respondió de manera efectiva al análisis de la situación problemática que es la guía de acción para poder generar una correcta planificación y formular los instrumentos de gestión de la institución educativa, empleando las estrategias para la formulación de la visión, misión, metas, valores, objetivos y actividades operativas alcanzables y realizables articulado con los demás instrumentos de gestión escolar, evaluado y sociabilizado con los actores educativos, garantizando de esta manera un trabajo eficiente.

Su cumplimiento y ejecución responsable recayeron en el equipo de planeamiento, directores y directivos que dirigen la gestión escolar; garantizando su consolidación de las actividades operativas, para una gestión escolar eficaz y eficiente que resuelve problemas emergentes, instrumentos de gestión escolar debidamente articulados; los resultados de estos indicadores nos demostrarán su logro o deficiencia. 


\section{Referencias Bibliográficas}

Loera, A. (2003). Gestión escolar centrada en el aprendizaje. Primera edición.

Matus, C. (1977), Planificación de situaciones. Fondo de cultura económica. México.

Martínez, M. (2004) Ciencia y arte en la metodología cualitativa. p. 240. Editorial Trillas México.

Ruiz, P. (2012). Aprendizaje, cultura y desarrollo una aproximación interdisciplinaria. Fondo editorial de la Pontificia Universidad Católica del Perú.

Vasilachis, I. (2006). Estrategias de Investigación Cualitativa. Editorial Gedisa. Biblioteca de educación.

Zambrano, A. (2006), Planificación estratégica, presupuesto y control de la Gestión Pública. 